See discussions, stats, and author profiles for this publication at: https://www.researchgate.net/publication/322755314

\title{
The Footloose Entrepreneur model with a finite number of equidistant regions
}

Technical Report · December 2017

DOI: $10.13140 /$ RG.2.2.10235.62241

\section{CITATIONS}

0

3 authors:

José Maria Gaspar

University of Lisbon

14 PUBLICATIONS 15 CITATIONS

SEE PROFILE

Joao Correia-da-Silva

University of Porto

52 PUBLICATIONS 348 CITATIONS

SEE PROFILE

Some of the authors of this publication are also working on these related projects:

Collusion View project

Project New Economic Geography View project
READS

77

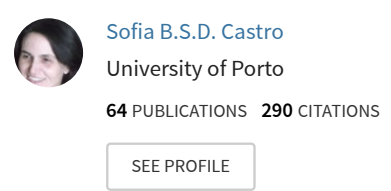




\title{
The Footloose Entrepreneur model with a finite number of equidistant regions
}

\author{
José Gaspar; Sofia B.S.D. Castro†, and João Correia-da-Silva
}

\begin{abstract}
We study the Footloose Entrepreneur model with a finite number of equidistant regions, focusing on the analysis of stability of three types of long-run equilibria: agglomeration, dispersion and partial dispersion. We find that, as the number of regions increases, there is more tendency for agglomeration and less tendency for dispersion. In the limit, as the number of regions tends to infinity, agglomeration becomes the unique stable equilibrium. Our conclusions are robust to any dependence of the total number of entrepreneurs and unskilled workers on the number of regions. Numerical evidence suggests that industry cannot disperse evenly among two regions when other regions have no industry. Finally, we introduce region heterogeneity in unskilled labour and obtain a more general condition for stability of agglomeration. We then study the impacts of regional asymmetries and find that having more unskilled workers in the core (or less in the periphery) increases the tendency for agglomeration.
\end{abstract}

Keywords: Core-Periphery, Footloose Entrepreneur, Finite number of regions, Agglomeration

JEL Classification Numbers: R10, R12, R23.

\section{Introduction}

The secular tendency for spatial agglomeration of economic activity is well known and has always been a matter of profound debate. Recent developments have allowed a more rig-

${ }^{*}$ CEF.UP and Faculty of Economics, University of Porto. Católica Porto Business School, Universidade Católica Portuguesa. Email: jgaspar.phd@fep.up.pt

${ }^{\dagger}$ CMUP and Faculty of Economics, University of Porto. Email: sdcastro@fep.up.pt

${ }^{\ddagger}$ CEF.UP and Faculty of Economics, University of Porto. E-mail: joao@fep.up.pt 
orous treatment of such phenomena, with recourse to microeconomic foundations. ${ }^{1}$ The benchmark in this literature is the Core-Periphery (CP) model, introduced by Krugman (1991). However, several issues have been raised in recent literature about possible shortcomings due to some simplifying assumptions. One of these shortcomings is the prevalent focus on the 2-region framework.

Theoretical insights on a model with three or more regions are interesting for different reasons. One reason is the understanding of interdependencies among many regions to guide empirical studies. A two-region set-up overlooks the variability of market access across regions (Fujita and Thisse, 2009), thus more complex impacts may arise in a multi-regional set-up compared to a two-region one (Fujita and Mori, 2005; Fujita and Thisse, 2009; Behrens and Robert-Nicoud, 2011). As pointed out by Fujita et al. (1999), the consideration of only two regions stems from the advantage of dealing with more tractable problems, although it seems implausible that the geographical dimension of economic activity can be reduced to a 2-region framework. It is important, therefore, to understand to what extent the main conclusions obtained using 2-region models extend to models with more regions.

This motivated a number of different studies. Castro et al. (2012) studied a 3-region version of the CP model by Krugman (1991), and also a version with $2 n$ regions equally spaced around a circle. Comparing the behaviour of the 3-region model relatively to the 2-region model, their main conclusion was that the additional region favours the agglomeration of economic activity and hinders the dispersion of economic activity. Akamatsu et al. (2012) sought to explain spatial agglomeration in a CP model with $2^{n}$ regions. They studied particular agglomeration patterns in the CP model as transportation costs steadily decrease over time, where the number of regions in which firms locate is reduced by half and the spacing between each pair of adjacent "core" regions doubles after each bifurcation. Oyama (2009) incorporated self-fulfilling expectations in migration decisions in a multi-regional variant of the CP model and studied global stability in just one core region in the presence of asymmetries or trade barriers. Tabuchi and Thisse (2011) studied the rise of a hierarchical system of central places in a multi-location space. Barbero and Zofío (2016) considered different network topologies in order to study how the interplay between centripetal and centrifugal forces change according to the heterogeneity of location space. They showed that more heterogeneous configurations enforce the likelihood of agglomeration in regions with higher centrality; i.e., those that are relatively better located. The role of heterogeneous distances between regions has also been addressed in other frameworks such as the racetrack economy (Krugman, 1993; Fujita et al., 1999;

\footnotetext{
${ }^{1}$ See Fujita et al. (1999), Ottaviano et al. (2002), Baldwin et al. (2003), Robert-Nicoud (2005) and the references therein.
} 
Picard and Tabuchi; 2010; Mossay, 2013), equally spaced regions on the line segment (e.g. Ago et al., 2006) or hexagonal distributions as in Ikeda et al. (2014). Tabuchi (2014), developed a multi-regional model based on Fujita et al. (1999) with exogenous asymmetries both in trade costs and in the distribution of the immobile workers and found that it is able to predict the historical tendency for agglomeration in the capital regions. The paper focused on extreme values of transportation costs (autarky and almost-free trade). Other recent contributions include Behrens et al. (2006), Akamatsu and Takayama (2009), Ikeda et al. (2012), Forslid and Okubo (2012), Commendatore et al. (2015a), Fabinger (2015). ${ }^{2}$

Some of the inherent technical difficulties that stem from the extension of a 2-region model to a multi-regional framework call for a base model that is more tractable than the original CP model (Krugman, 1991). An analytically solvable version of the CP model, dubbed the Footloose Entrepreneur (FE) model, was developed by Forslid and Ottaviano (2003). The only difference with respect to the original CP model is that, in the FE model, the variable input in the mobile sector is immobile labour instead of mobile labour. The role of mobile (footloose) labour becomes limited to the fixed input (entrepreneurship) in the mobile sector. This subtle modification renders the model analytically solvable because the marginal production cost becomes independent of the spatial distribution of economic activity.

This motivates us to consider an $n$-region version of the analytically solvable FE model as it allows us to obtain a closed-form solution for the regional utility level as a function of the spatial distribution of economic activity. ${ }^{3}$ Our set-up, by considering equidistant regions, allows us to characterize analytically the stability of equilibria. Equidistance among regions has been assumed in other works such as Tabuchi et al. (2005), Zeng and Uchikawa (2014) and Gaspar et al. (2017). The focus of Tabuchi et al. (2005) is on how the relationship between transport costs and congestion costs shapes the size of cities, which tend to increase in early stages and then shrink at later stages. ${ }^{4}$ Zeng and Uchikawa (2014) study spatial inequalities in a multi-region economy where each region differs in size and there is only one single good produced under monopolistic competition. In their setting, production is not footloose as the single input (capital) enters both in the fixed cost and in the variable cost of the firm. Gaspar et al. (2017) used a FE model but with a quasi-linear translog upper tier utility, as in Pflüger (2004), instead of the standard

\footnotetext{
${ }^{2}$ For a comprehensive and insightful overview of some of the main contributions concerning multiregional NEG models, see Commendatore et al. (2015b).

${ }^{3}$ Ottaviano et al. (2002) and Pflüger (2004) have also built analytically solvable CP models by considering quasi-linear preferences. These models are also potentially good candidates for extensions to multiple regions. In fact, the model of Pflüger (2004) was extended to the case of $n$ equidistant regions by Gaspar et al. (2017).

${ }^{4}$ Additionally, their setup differs from ours in the sense that a quasi-linear utility is assumed, as in Ottaviano et al. (2002).
} 
Cobb-Douglas form considered by Forslid and Ottaviano (2003).

Our main finding is that, as the number of regions increases, agglomeration becomes more likely while dispersion becomes less likely. More precisely, we conclude that: (i) the set of parameter values for which agglomeration is stable in an economy with $n$ regions is contained in the set of parameters for which it is stable in an economy with $n+1$ regions; and (ii) the set of parameter values for which dispersion is stable in an economy with $n+1$ regions is contained in the set of parameters for which it is stable in an economy with $n$ regions. This happens because, when unskilled workers are evenly distributed among regions, more regions implies less immobile consumers in each region and, therefore, lower local demand. As a result, a large region becomes relatively more attractive as the market-size effect becomes stronger relative to the market-crowding effect, which induces agglomeration.

We also consider a configuration of partial dispersion (i.e., symmetric dispersion across $m$ regions, with $m<n)$. Numerical simulations for $m=2$ and $n=3$ suggest that this configuration is always unstable, independently of parameter values. In addition, our simulations indicate that multiplicity of equilibria and locational hysteresis are a persistent feature of the FE model with an arbitrary number of regions, but disappears as $n$ tends to infinity because agglomeration becomes the only possible stable equilibrium.

As in Forslid and Ottaviano (2003), we introduce exogenous regional asymmetries in our $n$-region version of the FE model by considering regional heterogeneity in the endowment of unskilled labour across regions. By studying the stability conditions for agglomeration, we find that an increase of unskilled labour in the core region (or a decrease of unskilled labour in the peripheral regions) strengthens the tendency for agglomeration. This is achieved through an analysis of skilled labour wages providing further insight into the agglomeration mechanism for a finite number of regions.

We conclude that the impact of considering additional regions in the FE model is analogous to that of considering additional regions in the $\mathrm{CP}$ model. In this sense, the FE model behaves similarly to the CP model (as desired by its creators).

Our analysis focused on local stability of equilibria considering an arbitrary number of equidistant regions. It would be interesting to understand the interplay between variation in the number of regions and other factors shaping the space economy, such as the role of different spatial topologies studied by Barbero and Zofío (2016). This is left for future work.

The remainder of the paper is structured as follows. In Section 2, we obtain the general expressions for nominal and real wages as functions of the spatial distribution of the entrepreneurs. In Section 3, we address the dynamics of the model and find the stability conditions for three possible kinds of equilibria: agglomeration, total dispersion 
and partial dispersion. We also discuss how each of these outcomes becomes more or less likely as the parameters of the model change. In Section 4, we assess the effect of increasing the number of regions on the behaviour of the FE model. In Section 5, we allow for exogenous heterogeneity in the unskilled immobile labour factor in the FE model and determine a more general local stability condition for agglomeration in order to study how the spatial distribution of farmers is likely to influence the agglomerative outcome. In Section 6, we make some concluding remarks.

\section{Economic environment and short-run equilibrium}

The economy is composed by $n \geq 2$ regions that are assumed to be structurally identical and equidistant from each other. The framework is that of Forslid and Ottaviano (2003), except for the fact that an arbitrary number of regions is considered instead of only two. The total endowments of entrepreneurs and unskilled labour are, respectively, $H(n)$ and $L(n) .{ }^{5}$ Entrepreneurs can move among regions: $\sum_{i=1}^{n} H_{i}=H(n)$; while unskilled workers are immobile and assumed be evenly spread across the $n$ regions: $L_{i}=\frac{L(n)}{n}, \forall i$.

The representative consumer of region $i$ has utility:

$$
U_{i}=X_{i}^{\mu} A_{i}^{1-\mu}
$$

where $A_{i}$ is the consumption of agricultural products in region $i$ and $X_{i}$ is defined by the CES composite good:

$$
X_{i}=\left[\int_{s \in N} d_{i}(s)^{\frac{\sigma-1}{\sigma}} d s\right]^{\frac{\sigma}{\sigma-1}},
$$

where $d_{i}(s)$ is the consumption of variety $s$ of manufactures in region $i, N$ is the mass of existing varieties, and $\sigma>1$ is the constant elasticity of substitution between different varieties of manufactures, and $\mu \in(0,1)$ is the share of expenditure in manufactured goods.

Production requires, as inputs, $\alpha$ units of skilled labour (entrepreneurs) and $\beta$ units of unskilled labour for each unit that is produced. The production cost of a firm in region $i$ is:

$$
C_{i}\left(x_{i}\right)=w_{i} \alpha+w_{i}^{L} \beta x_{i},
$$

where $w_{i}$ is the nominal wage of skilled workers in region $i$ and $w_{i}^{L}$ is the nominal wage of unskilled workers in region $i$.

Trade of manufactures between two regions is subject to iceberg costs $\tau \in(1,+\infty)$.

\footnotetext{
${ }^{5}$ The dependence of these endowments on the number of regions increases the generality of the comparison between models with different numbers of regions.
} 
Let $\tau_{i j}$ denote the number of units that must be shipped at region $i$ for each unit that is delivered at region $j$. Since the regions are assumed to be equidistant from each other, we have the following trade cost structure:

$$
\begin{cases}\tau_{i j}=1, & \text { if } j=i \\ \tau_{i j}=\tau, & \text { if } j \neq i .\end{cases}
$$

The agricultural good is produced one for one using unskilled labour (under constant returns to scale) and is freely traded across regions. Let $p_{j i}(s)$ and $d_{j i}(s)$ denote the price and demand in region $i$ of a variety, $s$, that is produced in region $j$. Utility maximization yields the following regional demand:

$$
d_{j i}(s)=\frac{p_{j i}(s)^{-\sigma}}{P_{i}^{1-\sigma}} \mu Y_{i}
$$

where $P_{i}$ is the regional price index of manufactures, associated with (2):

$$
P_{i}=\left[\sum_{j=1}^{n} \int_{s \in N} p_{j i}(s)^{1-\sigma} d s\right]^{\frac{1}{1-\sigma}}
$$

and $Y_{i}$ is the regional income:

$$
Y_{i}=w_{i} H_{i}+w_{i}^{L} \frac{L(n)}{n}
$$

Turning to the supply side and starting with the agricultural sector, absence of transport costs implies that its price is the same everywhere $\left(p_{1}^{A}=\ldots=p_{n}^{A}\right)$. Furthermore, under perfect competition, we have marginal cost pricing: $p_{i}^{A}=w_{i}^{L}, \forall i$. Consequently, there is unskilled workers' wage equalization across regions: $w_{1}^{L}=\ldots=w_{n}^{L}$. Hence, by choosing the agricultural good as numeraire, we can set $p_{i}^{A}=w_{i}^{L}=1, \forall i$. We assume that the non-full-specialization (NFS) condition (Baldwin et al., 2003) holds, guaranteeing that agriculture is active in all regions even if all manufacturing activity takes place in a single region. ${ }^{6}$

Given the fixed cost in (3), the number of varieties manufactured in region $i$ is $v_{i}=$

\footnotetext{
${ }^{6}$ The NFS condition requires world expenditure on agricultural goods to be greater than the total production of agricultural goods in all regions except one, i.e., $(1-\mu) \sum_{i=1}^{n} Y_{i}>\frac{n-1}{n} L(n)$. This becomes $(1-\mu)\left[\sum_{i} w_{i} H_{i}+L(n)\right] \geq \frac{n-1}{n} L(n)$. For the particular distributions we are going to study, it can be shown that $\sum_{i} w_{i} H_{i}=\frac{\mu L}{(\sigma-\mu)}$, from which the NFS condition becomes $\mu<\frac{\sigma}{n(\sigma-1)+1}$.
} 
$H_{i} / \alpha$. A firm in region $i$ with total cost given by (3) maximizes the following profit:

$$
\pi_{i}(s)=\sum_{j=1}^{n} p_{i j}(s) d_{i j}(s)-\beta\left[\sum_{j=1}^{n} \tau_{i j} d_{i j}(s)\right]-\alpha w_{i} .
$$

The optimal price of firm $i$ is the same as in the 2-region model:

$$
p_{i j}(s)=\tau_{i j} \beta \frac{\sigma}{\sigma-1}
$$

All varieties produced in region $i$ are sold at the same price and are equally demanded in region $j$. Using (8), the CES price index (5) becomes:

$$
P_{i}=\alpha^{\frac{1}{\sigma-1}} \beta \frac{\sigma}{\sigma-1}\left(\sum_{j=1}^{n} \phi_{j i} H_{j}\right)^{\frac{1}{1-\sigma}}
$$

where $\phi_{i j} \equiv \tau_{i j}^{1-\sigma} \in(0,1)$ represents the "freeness of trade" between regions $i$ and $j$. Absence of entry barriers in the manufacturing industry translates into zero profits in equilibrium. Therefore, using (7) we reach:

$$
\alpha w_{i}=\sum_{j=1}^{n} p_{i j} d_{i j}-\beta \sum_{j=1}^{n} \tau_{i j} d_{i j}
$$

which becomes, considering the prices in (8):

$$
w_{i}=\frac{\beta x_{i}}{\alpha(\sigma-1)},
$$

where $x_{i} \equiv \sum_{j=1}^{n} \tau_{i j} d_{i j}$ is the total production by a manufacturing firm in region $i$. Using (4), (8) and (9), we obtain an expression for $x_{i}$ :

$$
x_{i}=\frac{\mu(\sigma-1)}{\alpha \beta \sigma} \sum_{j=1}^{n} \frac{\phi_{i j} Y_{i}}{\sum_{m=1}^{n} \phi_{m j} v_{m}} .
$$

Replacing (11) in (10), and given that $v_{i}=H_{i} / \alpha$, we obtain:

$$
w_{i}=\frac{\mu}{\sigma} \sum_{j=1}^{n} \frac{\phi_{i j} Y_{j}}{\sum_{m=1}^{n} \phi_{m j} H_{m}} .
$$

From (6), regional income equals:

$$
Y_{i}=\frac{L(n)}{n}+w_{i} H_{i}
$$


The spatial distribution of entrepreneurs can be described, in relative terms, by the vector $h \equiv\left(h_{1}, \ldots, h_{n}\right)$, where $h_{i} \equiv \frac{H_{i}}{H(n)}, \forall i$. The fraction of entrepreneurs in region $n$ may be omitted because it is implicit in the other fractions: $h_{n}=1-\sum_{i=1}^{n-1} h_{i}$. The price index of manufactures, $P_{i}$, becomes, from (9):

$$
P_{i}(h)=\frac{\beta \sigma}{1-\sigma}\left(\frac{\alpha}{H(n)}\right)^{\frac{1}{\sigma-1}}\left(\sum_{j=1}^{n} \phi_{j i} h_{j}\right)^{\frac{1}{1-\sigma}} .
$$

Using (12) and (13), we obtain a system of $n$ equations that determines the nominal wages in each region:

$$
w_{i}=\frac{\mu}{\sigma} \sum_{j=1}^{n} \frac{\phi_{i j}\left[\frac{L(n)}{n H(n)}+w_{j} h_{j}\right]}{\sum_{m=1}^{n} \phi_{m j} h_{m}} .
$$

For illustrative purposes, we present the closed-form solution for the equilibrium nominal wage of entrepreneurs as a function of their spatial distribution for $n=3:^{7}$

$$
w_{i}=\frac{\frac{\mu L}{3 \sigma H(n)}\left[\sum_{j=1}^{3} \frac{\phi_{i j}}{r_{j}}+\frac{\mu}{\sigma}\left(\phi(\phi-1) \frac{\sum_{k \neq i} h_{k}}{\prod_{k \neq i} r_{k}}+\frac{\phi^{2}-1}{r_{i}} \sum_{k \neq i} \frac{h_{k}}{r_{k}}\right)+\frac{\mu^{2}}{\sigma^{2}}\left(2 \phi^{3}-3 \phi^{2}+1\right) \frac{1}{r_{i}} \prod_{k \neq i} \frac{h_{k}}{r_{k}}\right]}{1-\frac{\mu}{\sigma} \sum_{j=1}^{3} \frac{h_{j}}{r_{j}}+\frac{\mu^{2}}{\sigma^{2}}\left(1-\phi^{2}\right)\left(\frac{h_{1} h_{2}}{r_{1} r_{2}}+\frac{h_{1} h_{3}}{r_{1} r_{3}}+\frac{h_{2} h_{3}}{r_{2} r_{3}}\right)-\frac{\mu^{3}}{\sigma^{3}}\left(2 \phi^{3}-3 \phi^{2}+1\right) \prod_{j=1}^{3} \frac{h_{j}}{r_{j}}},
$$

where $r_{j} \equiv \sum_{m=1}^{3} \phi_{m j} h_{m}$.

Since agents spend a fraction $\mu$ of their income on manufactures, whose price index is $P_{i}$, and the price of agricultural goods is unity, the real wage or indirect utility level of entrepreneurs is given by:

$$
\omega_{i}=w_{i} / P_{i}^{\mu}
$$

\section{Long-run equilibria and stability}

Entrepreneurs tend to migrate to the region that offers them the highest real wage or indirect utility (17). For concreteness, we consider replicator dynamics: the flow of entrepreneurs to a region is proportional to the difference between the region's real wage and the weighted average real wage and to the number of entrepreneurs in the region.

Formally, the dynamics are described by the following system of $n-1$ ordinary differ-

\footnotetext{
${ }^{7}$ See Appendix A for details on its calculation.
} 
ential equations defined in the simplex $\Delta \equiv\left\{h \in \mathbb{R}_{+}^{n}: \sum_{i=1}^{n} h_{i}=1\right\}:^{8}$

$$
\left\{\begin{array}{l}
\dot{h}_{1}=\left(\omega_{1}-\bar{\omega}\right) h_{1} \\
\ldots \\
\dot{h}_{n-1}=\left(\omega_{n-1}-\bar{\omega}\right) h_{n-1}
\end{array} \quad, \quad h_{i} \in[0,1], \quad \forall i \in\{1, \ldots, n-1\}\right.
$$

where $\bar{\omega} \equiv \sum_{i=1}^{n} h_{i} \omega_{i}$ is the weighted average real wage. Migration to the omitted region in (18) is, consistently, given by $\dot{h}_{n}=-\sum_{i=1}^{n-1} \dot{h}_{i}=\left(\omega_{n}-\bar{\omega}\right) h_{n}$.

In this dynamical system, migration to empty regions has to be started exogenously. If a region is empty, $h_{i}=0$, then the corresponding differential equation for the dynamics yields $\dot{h}_{i}=0$, which means that the region remains empty. Hence, the boundary of the simplex is invariant for the dynamics.

Direct substitution in equations (18) shows that the configurations:

$$
h \in\left\{(1,0, \ldots, 0),\left(\frac{1}{n}, \ldots, \frac{1}{n}\right),\left(\frac{1}{m}, \ldots, \frac{1}{m}, 0, \ldots, 0\right)\right\}
$$

and their permutations are equilibria. The equilibria represented by $(1,0, \ldots, 0)$ and its permutations correspond to full agglomeration of industry in one region. This outcome is called agglomeration or concentration. The second configuration describes an even distribution of industry among the $n$ regions. This outcome is called total dispersion. The last configuration represents an even distribution of industry among only $m$ of the $n$ regions (with $m<n$ ), while the remaining regions are deprived of industry. This is what we call partial dispersion. The expression "partial dispersion" reflects that not all regions have industry (partial) and that there are entrepreneurs in more than one region (dispersion). Stability of each equilibrium is preserved by permutation so that the same stability conditions hold for agglomeration or partial dispersion in any of the regions.

Definition 1. An equilibrium is said to be stable if, after any small exogenous migration of skilled workers across regions, the spatial distribution of skilled workers is pulled back to the initial one.

\subsection{Stability of total dispersion}

Since total dispersion is an interior configuration, its stability is given by the sign of the real part of the eigenvalues of the Jacobian matrix of the system in $(18)$ at $h=\left(\frac{1}{n}, \ldots, \frac{1}{n}\right)$. This matrix has a repeated real eigenvalue with multiplicity $n-1$, which is given by

\footnotetext{
${ }^{8}$ Note that, even though the spatial distribution of entrepreneurs can be described using $n-1$ coordinates, we sometimes use all $n$ coordinates for the sake of clarity.
} 
(see Appendix B):

$$
\frac{\partial \omega_{i}}{\partial h_{i}}\left(\frac{1}{n}, \ldots, \frac{1}{n}\right)
$$

Total dispersion is stable if (19) is negative, that is, differentiating (17), dispersion is stable if:

$$
\frac{\partial w_{i} / \partial h_{i}}{w_{i}}<\frac{\partial P_{i}^{\mu} / \partial h_{i}}{P_{i}^{\mu}}
$$

where $P_{i}^{u}$ is the regional price index. This means that the stability of dispersion can be described in terms of semi-elasticities. Entrepreneurs remain equally dispersed across the $n$ regions if a migration of entrepreneurs to a region induces a percentage change in the nominal wage smaller than the corresponding percentage change in prices. In this case, the loss in purchasing power due to an increase of the share of entrepreneurs, $h_{i}$, leads to an exodus from that region until the initial share of entrepreneurs is restored, that is, until $h_{i}=\frac{1}{n}$.

Proposition 1. Total dispersion is stable if: ${ }^{9}$

$$
B P(\phi) \equiv \mu(2 \sigma-1)[(n-1) \phi+1]-\mu^{2}(1-\phi)-(\sigma-1) \sigma(1-\phi)<0 .
$$

Proof. This is a sufficient condition for the eigenvalue in (19) to be negative. See Appendix B.

To rule out the possibility that $B P(\phi)>0, \forall \phi \in(0,1)$, which would preclude stability of total dispersion, we assume:

$$
\sigma>\mu+1
$$

commonly referred to as the no black-hole condition. ${ }^{10}$ Notice that $B P(\phi)$ is a linear function of $\phi$ with positive coefficient. Equating $B P(\phi)$ to zero and solving for $\phi$ gives:

$$
\phi_{b} \equiv \frac{(\sigma-\mu)(\sigma-1-\mu)}{(\sigma-\mu)(\sigma-1-\mu)+n \mu(2 \sigma-1)}<1
$$

which is the threshold level for $\phi$ below which (20) holds and thus total dispersion is stable. Lower transportation costs (higher $\phi$ ) discourage dispersion. Following Fujita et al. (1999), we call $\phi_{b}$ the break point. Given the no black-hole condition in (21), we have $\phi_{b}>0$, meaning that there always exists a freeness of trade below which dispersion is stable (given the values of $\mu$ and $\sigma$ ). On the other hand, the fact that $\phi_{b}<1$ means that there always exists a level of freeness of trade above which dispersion is unstable.

\footnotetext{
${ }^{9}$ If $B P(\phi)>0$, total dispersion is unstable. If $B P(\phi)=0$, total dispersion may be stable or unstable. See Tabuchi et al. (2005), footnote 10 .

${ }^{10}$ If agents have a very strong preference for variety $(\sigma<1+\mu)$, total dispersion is never stable.
} 


\subsection{Stability of agglomeration}

Agglomeration, $\left(h_{1}, h_{2}, \ldots, h_{n}\right)=(1,0, \ldots, 0)$, is a corner solution on a vertex of the simplex. Given the absence of entrepreneurs in all regions except one, the weighted average real wage is simply $\bar{\omega}=\omega_{1}$, that is, the weighted average real wage is the same as the real wage in region 1.

By symmetry, the real wages in the empty regions are equal $\left(\omega_{2}=\ldots=\omega_{n}\right)$. That $\omega_{1}>\omega_{2}$ is sufficient for agglomeration to be stable follows from the fact that entrepreneurs migrate to regions with higher real wages, together with continuity of real wages with respect to the spatial distribution. If the indirect utility of entrepreneurs is strictly higher in region 1 than in the all the other regions, there are no incentives for entrepreneurs in region 1 to migrate. We can say that agglomeration in region 1 is a stable configuration if: ${ }^{11}$

$$
\begin{gathered}
\omega_{1}>\omega_{2} \Longleftrightarrow \\
\frac{w_{1}}{P_{1}^{\mu}}>\frac{w_{2}}{P_{2}^{\mu}} \Leftrightarrow \frac{w_{1}}{w_{2}}>\left(\frac{P_{1}}{P_{2}}\right)^{\mu} \Leftrightarrow \frac{w_{1}}{w_{2}}>\phi^{\frac{\mu}{\sigma-1}} .
\end{gathered}
$$

Since $\phi^{\frac{\mu}{\sigma-1}}<1$, a sufficient condition for the stability of agglomeration is that the nominal wage is higher in the core than in the periphery. From the expression for $w_{1}$ and $w_{2}$ in (15), the next proposition is obtained by rearranging the expression $S P(\phi) \equiv w_{2} \phi^{\frac{\mu}{\sigma-1}}-w_{1}$.

Proposition 2. Agglomeration is a stable equilibrium if: ${ }^{12}$

$$
S P(\phi) \equiv \sigma-\mu+[\sigma+\mu(n-1)] \phi^{2}+[\sigma(n-2)+\mu(2-n)] \phi-n \sigma \phi^{1-\frac{\mu}{\sigma-1}}<0 .
$$

Since $S P(\phi)$ is a convex function and we have $S P(0)>0, S P(1)=0$ and $S P^{\prime}(1)>0$, the function $S P(\phi)$ has exactly one zero in $\phi \in(0,1)$. This value, denoted $\phi_{s}$, is the sustain point, i.e., the threshold for $\phi$ above which agglomeration is a stable equilibrium. If the "freeness of trade" parameter is high enough (i.e., if transport costs are low enough), agglomeration is stable. This is because lower transport costs imply that price indices become relatively higher in the regions that are deserted and thus real wages become relatively lower.

\footnotetext{
${ }^{11}$ It is unstable if $\omega_{1}<w_{2}$.

${ }^{12}$ It is unstable if $S P(\phi)>0$.
} 


\subsection{Stability of partial dispersion}

We now address the stability of configurations in which entrepreneurs are equally dispersed across $m$ regions, with $1<m<n$, while the remaining $n-m$ regions are empty. Such configurations are always equilibria of the dynamical system (18). For simplicity, we focus on the possibility of dispersion across two regions, i.e., of the configuration $h^{p} \equiv$ $h=\left(\frac{1}{2}, 0, \ldots, 0, \frac{1}{2}\right)$ and its permutations.

Proposition 3. If partial dispersion between two regions is stable, then $\gamma \geq 0$ and $\Psi \leq 0$, where:

$$
\left\{\begin{array}{l}
\gamma \equiv \sigma(1+\phi)\left[1-\phi^{1-\frac{\mu}{\sigma-1}}\left(\frac{1+\phi}{2}\right)^{\frac{\mu}{\sigma-1}}\right] n-[2 \sigma+\mu(n-2)](1-\phi)(2 \phi+1) . \\
\Psi \equiv \mu(\sigma+3 \sigma \phi-2 \phi) n-\mu^{2}(1-\phi) n-\mu(1-\phi)-2 \sigma(\sigma-1-\mu)(1-\phi)
\end{array}\right.
$$

Proof. See Appendix C.

Partial dispersion appears to be unstable for all parameter values. However, because of the non-linearity of $\gamma(\phi)$, we are not able to prove this analytically. Numerical inspection of both conditions in (24) suggests that these are never simultaneously met, as illustrated in Figure 1 for the case of three regions. This conclusion is not surprising, since partial dispersion has also been numerically shown to be unstable in other models, such as the 3-region CP model by Castro et al. (2012) and by Fujita et al. (1999).

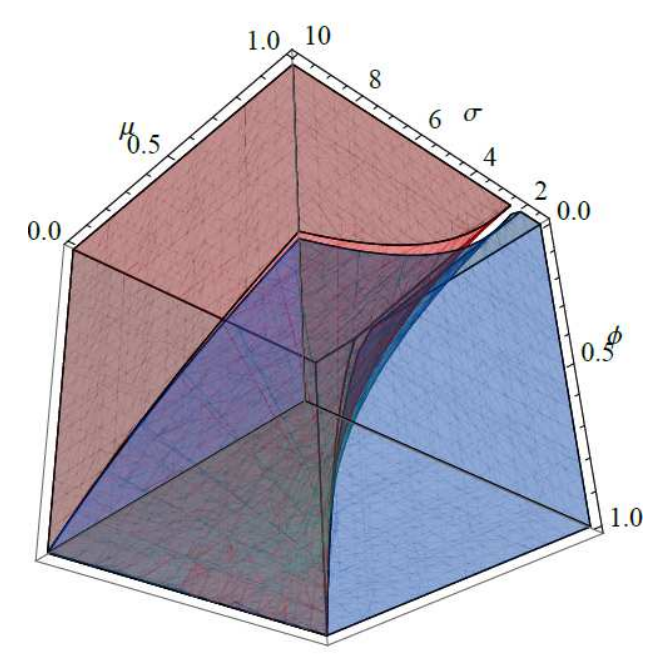

Figure 1 - Parameter regions in which $\gamma \geq 0$ and $\Psi \leq 0$ for $n=3$. The two regions do not seem to overlap, indicating that the two stability conditions are never simultaneously satisfied.

The consideration of a greater number of regions seems to reinforce this conclusion. The no black-hole condition implies that the derivative of $\Psi$ with respect to $n$ is positive. 
Note that $\frac{\partial \gamma}{\partial n}>0$. However, it is $\frac{\partial \Psi}{\partial n}>0$ that suggests the instability of partial dispersion. This leads to the conjecture that partial dispersion between two regions is never stable for any $n .^{13}$

\section{Impact of the number of regions}

A reason to build an $n$-region model is to be able to understand the impact of the number of regions on the behaviour of the FE model. Castro et al. (2012) proved that, in an extension of Krugman's CP model to three regions, more regions favour agglomeration as a stable outcome. Here, we obtain a stronger result in the same direction.

Proposition 4. The parameter region for which agglomeration is stable in the FE model with $n+1$ regions contains that of the FE model with $n$ regions. Comparing agglomeration between both models, we find that: (i) the ratio between price indices in the core and in the periphery are the same; (ii) the ratio between nominal wages in the core and periphery is higher in the model with $n+1$ regions.

Proof. See Appendix D.

In other words, an increase in the number of regions favours stability of agglomeration in the FE model. We next detail the explanation for why this happens.

An entrepreneur who migrates to the periphery will find a cost-of-living that is independent of the number of regions, but the size of the internal market is smaller compared to the core if there are more regions because the fraction of unskilled workers that live there is smaller. All the other entrepreneurs and the remaining unskilled workers would constitute the external market. Given the existence of transportation costs to the other regions, this entrepreneur will face a lower relative global demand in a model with more regions, and, therefore, will earn a lower nominal wage. This leads to the fact that agglomeration is more likely in a model with more regions. Recalling expression (23) from Proposition 4, it can be shown that $\lim _{n \rightarrow+\infty} S P(\phi)<0$, which means that, in the limit, when the number of regions tends to infinity, agglomeration is always stable.

We are also able to compare the stability conditions of total dispersion in the FE model with $n$ regions and the FE model with $n+1$ regions.

Proposition 5. The parameter region for which total dispersion is stable in the FE model with $n$ regions contains that of the FE model with $n+1$ regions.

\footnotetext{
${ }^{13}$ This result is confirmed analytically in the multi-region setting by Gaspar et al. (2017). However, their work is based on quasi-linear upper tier utility instead of Cobb-Douglas and is therefore less related to the original CP model than our framework.
} 
Proof. Observe that $\phi_{b}$ in (22) decreases as the number of regions increases. This means that an increase in the number of regions makes total dispersion less likely to be stable.

We conclude that an increase in the number of regions hinders stability of total dispersion. After a small deviation from total dispersion, the market-size effect and the market-crowding effect in the larger region becomes stronger (resp. weaker) as the number of regions increases, because each of the smaller regions individually faces a lower local demand given less unskilled workers. Therefore, as the number of immobile workers per region decreases, so diminishes its role as a dispersive force. From (20) in Proposition 2 , it is possible to verify that $\lim _{n \rightarrow+\infty} B P(\phi)>0$. Thus, in the limit, when the number of regions tends to infinity, total dispersion is never stable. In this case, the amount of immobile workers in each region becomes negligible.

Remark. Notice that stability of agglomeration or dispersion does not depend on the total number of entrepreneurs and unskilled workers. Therefore, our conclusions are robust to any possible dependence of the global size and composition of the workforce on the number of regions.

In the equidistant multi-regional model by Gaspar et al. (2017), who use quasi-linear utility as in Pflüger (2004), it is possible to show that more regions also favour full agglomeration and discourage total dispersion, but only for a fixed global unskilled to skilled labour ratio $L / H$, i.e., the impact of more regions depends on how the global composition of workers changes with $n .^{14}$

\subsection{Numerical evidence of locational hysteresis}

One feature of the 2-region CP and FE models is the existence of a subcritical pitchfork bifurcation. This kind of bifurcation implies that there are values of the freeness-of-trade parameter, $\phi \in\left(\phi_{s}, \phi_{b}\right)$, for which total dispersion and agglomeration are simultaneously stable. Therefore, the long-run distribution of economic activity depends on the initial distribution of economic activity. The model exhibits locational hysteresis.

Recall from (20) and (23) that $B P$ and $S P$ are functions of $(\phi, \sigma, \mu, n)$. We establish analytically the existence of an open subset in parameter space $(\phi, \sigma, \mu, n)$ in which both total dispersion and agglomeration are stable outcomes. Consider the point in parameter space $(\phi, \sigma, \mu, n)=(0.4,3,0.4,3)$. At this point, we have both $B P<0$ and $S P<0$. Therefore, both total dispersion and agglomeration are stable equilibria. Since $B P$ and $S P$ are continuous functions of $(\phi, \sigma, \mu, n)$, they remain negative in an open neighbourhood of $(0.4,3,0.4,3)$

\footnotetext{
${ }^{14}$ It would be interesting to further investigate this result in a different setting, like an extension of Ottaviano et al. (2002) to incorporate equidistant multiple regions.
} 
Simulating the expressions for $B P$ and $S P$ in (20) and (23) for a grid of values of $\mu$ and/or $\sigma$, we always obtain an interval for $\phi$ where we have simultaneity of agglomeration and dispersion. Our results are displayed in Figure 2. The picture to the left depicts
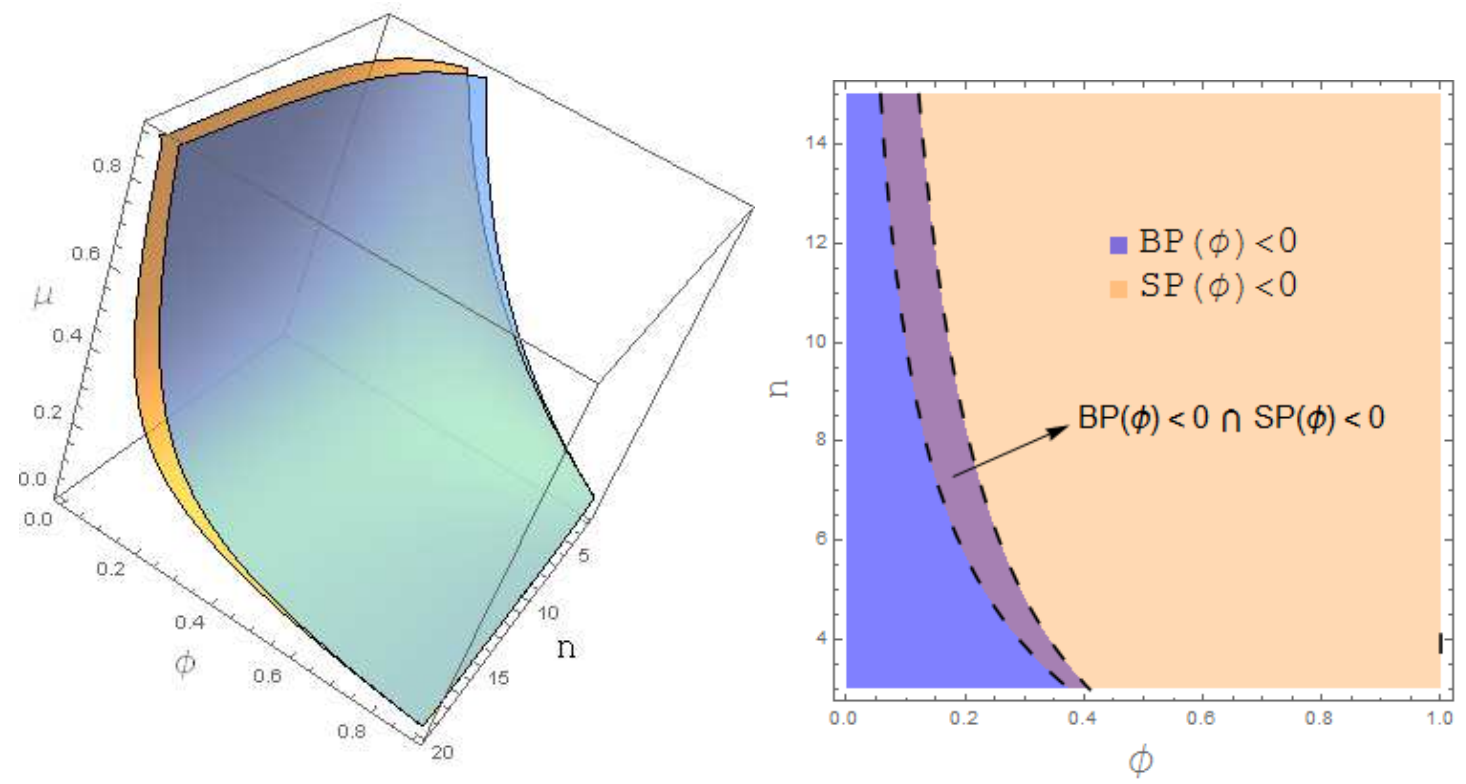

Figure 2 - To the left we have $\{(\phi, \sigma \mu, n): B P=0\} \cup\{(\phi, \sigma \mu, n): S P=0\}$ for $\sigma=3$. To the right, we have $\{(\phi, \sigma \mu, n): B P<0\} \cup\{(\phi, \sigma \mu, n): S P<0\}$ for $\sigma=3$ and $\mu=0.3$.

the surfaces corresponding to $B P(\phi, 3, \mu, n)=0$ and $S P(\phi, 3, \mu, n)=0$ in $(\phi, \mu, n)$ space. ${ }^{15}$ Between the surfaces we have $\{(\phi, \sigma \mu, n): B P<0\} \cap\{(\phi, \sigma \mu, n): S P<0\}$, so agglomeration and dispersion are both stable. Although this region in $(\phi, n, \mu)$ space appears to be very thin, it suggests that, for any triple $(\sigma, n, \mu)$, there always exists values of $\phi$ for which agglomeration and dispersion are simultaneously stable. Thus $\phi_{s}<$ $\phi_{b}$ and there is locational hysteresis. ${ }^{16}$ To the right, we fix $\mu$ and portray the planes $\{(\phi, n): B P(\phi, 3,0.3, n)<0\} \cup\{(\phi, n): S P<0(\phi, 3,0.3, n)\}$. The region between the dashed lines corresponds to $\{(\phi, n): B P(\phi, 3,0.3, n)<0\} \cap\{(\phi, n): S P<0(\phi, 3,0.3, n)\}$, which implies simultaneity of agglomeration and total dispersion. This picture shows strong evidence that this simultaneity always exists whatever the number of regions. Moreover, the scope for simultaneity, measured by the difference $\phi_{b}-\phi_{s}$, seems to be increasing in $n$. In other words, more regions imply a greater range of values of the freeness of trade $\phi$ for which agglomeration and dispersion are simultaneously stable.

Our evidence suggests that, for any given values of $n, \mu$ and $\sigma$, there always exists value of $\phi$ for which total dispersion and agglomeration simultaneously stable, i.e., we

\footnotetext{
${ }^{15}$ The choice to fix $\sigma$ was due to the fact that our simulations evidenced invariance of the results in the choice of values for this parameter.

${ }^{16}$ It is worth stressing the fact that our evidence suggests invariance of our results to changes in $\sigma$.
} 
always have $\phi_{s}<\phi_{b}$. This means that the $n$-region FE model also exhibits locational hysteresis. In this sense, temporary decreases in transport costs below the sustain point may trigger agglomeration in one region permanently.

\section{Agglomeration under asymmetric regions}

In this section we let the regions differ in terms of their endowments of farmers. As a result, we are introducing exogenous regional differences such that now each $L_{i}$ may be different from $L_{j}, i \neq j$. Without loss of generality, assume that all industry is concentrated in region 1 . From (14), regional price indices remain unchanged compared to the symmetric case:

$$
P_{1}=\frac{\beta \sigma}{1-\sigma}\left(\frac{\alpha}{H(n)}\right)^{\frac{1}{\sigma-1}}, \text { and } P_{i \neq 1}=\frac{\beta \sigma}{1-\sigma}\left(\frac{\alpha}{H(n) \phi}\right)^{\frac{1}{\sigma-1}} .
$$

Nominal wages are determined by (12) and by the adapted version of (13), $Y_{i}=L_{i}+$ $w_{i} H_{i}, \forall i$. Agglomeration is stable if $\omega_{1}>\max _{i \neq 1}\left\{\omega_{i}\right\}$. Since regional prices remain unchanged, agglomeration in region 1 is then stable if:

$$
\frac{w_{i}}{\max _{i \neq 1}\left\{w_{i}\right\}}>\left(\frac{P_{1}}{P_{i \neq 1}}\right)^{\mu} \Longleftrightarrow \frac{w_{i}}{\max _{i \neq 1}\left\{w_{i}\right\}}>\phi^{\frac{\mu}{\sigma-1}}
$$

Assume also w.l.o.g. that region 2 is the region among the $n-1$ peripheral regions that has highest nominal wage, i.e., $w_{2} \geq w_{i}, \forall i \neq 1$. Then agglomeration is stable if:

$$
\frac{w_{1}}{w_{2}}>\phi^{\frac{\mu}{\sigma-1}} \text {. }
$$

This leads to the following result.

Proposition 6. Agglomeration in region 1 is stable under exogenous asymmetries if:

$$
\Gamma \equiv \phi^{\frac{\mu}{\sigma-1}}\left\{\sigma \phi^{2} L_{1}+L_{2}\left[\sigma-\mu\left(1-\phi^{2}\right)\right]+\phi \sum_{j=3}^{n} L_{j}[\sigma-\mu(1-\phi)]\right\}-\sigma \phi L(n)<0,
$$

and $L_{1} \geq L_{2}$. In this case, agglomeration in region 1 is stable if the freeness of trade is high enough.

Proof. See Appendix E.

If $L_{1} \geq L_{2}$, there exists a sustain point $\phi_{s} \equiv \phi \in(0,1)$ above which agglomeration in region 1 is stable. However, if $L_{1}<L_{2}$ it is possible that agglomeration in region 1 is never stable even as trade barriers approach zero. Such an outcome would require the number of farmers in region 1 relative to region 2 to be very low. 
By looking at nominal wages, we explain in detail how the distribution of farmers across different regions impacts the likelihood of agglomeration. First, from Appendix E, we get the entrepreneur's nominal wage at the core region:

$$
w_{1}=\frac{\mu\left(L_{1}+\sum_{j=3}^{n} L_{j}+L_{2}\right)}{H(n)(\mu-\sigma)} .
$$

The entrepeneur's nominal and real wage at the core is the same as in the case of symmetric regions. In the numerator, we rewrote $L(n)$ as the sum of the farmers in region 1 with the farmers in region 2 and the farmers living in all the other regions. An increase in the number of farmers in any of the regions impacts the core's nominal wage with the same positive magnitude. The wage in region 2 (see Appendix E) is given by:

$$
w_{2}=\frac{\mu\left\{L_{1} \sigma \phi^{2}+L_{2}\left[\sigma-\mu(1-\phi)^{2}\right]+\sum_{j=3}^{n} L_{j} \phi[\sigma-\mu(1-\phi)]\right\}}{H(n) \sigma \phi(\sigma-\mu)}
$$

Remark. Since $\sigma-\mu\left(1-\phi^{2}\right)>\phi[\sigma-\mu(1-\phi)]$, it follows that $w_{2}=\max _{i \neq 1}\left\{w_{i}\right\}$ if and only if $L_{2} \geq L_{i}, \forall i \neq 1$. Therefore, the periphery with the highest nominal wage corresponds to the periphery that has the highest number of farmers.

From inspection of (27), an increase in the number of farmers in any region also increases $w_{2}$. However, the marginal impact of $L_{2}$ on $w_{2}$ is higher than that of each $L_{j \neq 1,2}$, which, in turn, is higher than that of $L_{1}$. Using (26) and (27):

$$
\frac{w_{1}}{w_{2}}=\frac{\left(L_{1}+\sum_{j=3}^{n} L_{j}+L_{2}\right) \sigma \phi}{L_{2}\left[\sigma-\mu\left(1-\phi^{2}\right)\right]+\sum_{j=3}^{n} L_{j} \phi[\sigma-\mu(1-\phi)+\sigma]+L_{1} \sigma \phi^{2}} .
$$

The wage ratio is increasing in $L_{1}$, but decreasing in $L_{2}$ and any $L_{j \neq 1,2}$. This means that agglomeration is more (less) likely if $L_{1}$ (resp. $L_{2}, L_{j \neq 1,2}$ ) increases. Moreover, the negative impact of $L_{2}$ on is higher than that of $L_{j \neq 1,2}$. The economic intuition does not stray from that given in Section 4. In fact, the present case encompasses the symmetric one, as an increase in $L_{1}$ or a decrease in $L_{2}$ decreases the relative nominal (and real) wage in region 2. Analogous reasoning in the symmetric case would be to consider an increase in the number of regions and resulting decrease in a periphery's $L_{i}=L / n$. Both cases lead to a strengthening of the agglomerative outcome.

\section{Conclusion}

Building on the 2-region FE model by Forslid and Ottaviano (2003), we have obtained both analytical and numerical results from a FE model with an arbitrary number of 
equidistant regions. We have shown that an increase in the number of regions, other things being equal, favours stability of agglomeration and hinders stability of total dispersion. This happens because the amount of immobile unskilled workforce per region decreases which in turn diminishes its role as a dispersive force. In the limit, when the number of regions tends to infinity, agglomeration becomes the unique stable equilibrium.

Upon introducing exogenous asymmetries in the regional distribution of unskilled labour, we have concluded that market size effects operate through the nominal (hence real) wages in a way such that an increase in unskilled labour in the core region strengthens the stability of agglomeration. The same happens if unskilled labour in the peripheral regions is decreased. This is what actually happens in the symmetric case when the number of regions increases.

We have also provided numerical evidence that strongly suggests that dispersion of entrepreneurs between two regions is never stable in a model with more than two regions, where it corresponds to an outcome of partial dispersion.

Finally, we have found numerical evidence in that, for every triple $(\mu, \sigma, n)$, there exists $\phi \in(0,1)$ where agglomeration and total dispersion can be simultaneously stable, though this outcome is relatively unlikely. This means that, like the 2-region model, the FE model with more regions exhibits a core-periphery pattern based on a "subcritical pitchfork" bifurcation. The scope for multiplicity of equilibria seems to be higher for a higher number of regions.

These results are in the same direction as those obtained by Castro et al. (2012) for the CP model with three regions. Through the more tractable framework of the FE model we were able to obtain explicit solutions for the wages of entrepreneurs and show that most key features of Krugman's seminal CP model are likely to hold in a multi-regional set-up.

Further developments of our analysis could benefit from the interplay between the number of regions and other key variables. For instance, letting the number of regions and transportation costs co-vary could help us compare agglomeration patterns across different jurisdiction levels (e.g., country, province, county, city). Additionally, the impact of the number of regions could depend on (or influence) the relative location of regions in space. This means studies which have focused on the role of space heterogeneity under a limited number of regions (e.g., Barbero and Zofío, 2016) may be complementary to our own.

Acknowledgements We thank Paulo Guimarães, Vasco Leite, Pascal Mossay, Cesaltina Pires and José P. Pontes for very useful comments and suggestions. We also thank the participants in the $2^{\text {nd }}$ Ph.D. Workshop on Industrial and Public Economics at U. Rovira 
i Virgili and in the $3^{\text {rd }}$ Industrial Organization and Spatial Economics conference at Saint Petersburg. José Gaspar gratefully acknowledges support from CEF.UP. This research was financed by the European Regional Development Fund through COMPETE 2020 Programa Operacional Competitividade e Internacionalização (POCI) and by Portuguese Public Funds through Fundação para a Ciência e Tecnologia in the framework of projects POCI-01-0145-FEDER-006890, PEst-OE/EGE/UI4105/2014, PEst-C/MAT/UI0144/2013, and Ph.D. scholarship SFRH/BD/90953/2012. Part of this work was developed while João Correia-da-Silva was a Marie Curie Fellow at Toulouse School of Economics, financed by the European Commission (H2020-MSCA-IF-2014-657283).

\section{References}

Ago, T., Isono, I., Tabuchi, T. (2006) "Locational disadvantage of the hub", Annals of Regional Science, 40 (4), 819-848.

Akamatsu, T., Takayama, Y. (2009), "A simplified approach to analyzing multi-regional core-periphery models", Technical report, University Library of Munich.

Akamatsu, T., Takayama, Y., Ikeda, K. (2012), "Spatial Discounting, Fourier, and Racetrack Economy: A Recipe for the Analysis of Spatial Agglomeration Models", Journal of Economic Dynamics \& Control, 36, 1729-1759.

Baldwin, R., Forslid, R., Martin, P., Ottaviano, G., Robert-Nicoud, F. (2003), Economic Geography and Public Policy, Princeton University Press.

Barbero, J., Zofío, J. L. (2016), "The multiregional core-periphery model: The role of the spatial topology", Networks and Spatial Economics, 16 (2), 469-496.

Behrens, K., Gaigne, C., Ottaviano, G. I., Thisse, J.-F. (2006), "Is remoteness a locational disadvantage?", Journal of Economic Geography, 6(3), 347-368.

Behrens, K., Robert-Nicoud, F. (2011), “Tempora mutantur: In search of a new testament for NEG", Journal of Economic Geography, 11 (2), 215-230.

Castro, S.B.S.D., Correia-da-Silva, J., Mossay, P. (2012), "The Core-Periphery model with three regions and more", Papers in Regional Science, 91 (2), 401-418.

Commendatore, P., Kubin, I., Sushko, I. (2015a), "Typical bifurcation scenario in a three regional identical New Economic Geography model", Mathematics and Computers in Simulation, 108, 63-80. 
Commendatore, P., Filoso, V., Grafeneder-Weissteiner, T., Kubin, I. (2015b), "Towards a multiregional NEG framework: comparing alternative modelling strategies, in Complexity and Geographical Economics, 13-50, Springer.

Fabinger, M. (2015), "Cities as solitons: Analytic solutions to models of agglomeration and related numerical approaches", available at SSRN 2630599.

Forslid, R., Ottaviano, G. (2003), "An Analytically Solvable Core-Periphery Model", Journal of Economic Geography, 3, 229-240.

Forslid, R., Okubo, T. (2012), "On the development strategy of countries of intermediate size - an analysis of heterogeneous firms in a multi-region framework", European Economic Review, 56(4), 747-756.

Fujita, M., Krugman, P., Venables, A. (1999), The Spatial Economy: Cities, Regions and International Trade, Cambridge, MA: MIT Press.

Fujita, M., Mori,T. (2005), "Frontiers of the New Economic Geography", Papers in Regional Science, 84 (3), 377-405.

Fujita, M., Thisse, J.-F. (2009), "New Economic Geography: An appraisal on the occasion of Paul Krugman's 2008 Nobel Prize in Economic Sciences", Regional Sciences and Urban Economics, 39 (2), 109-119.

Gaspar, J., Castro, S.B.S.D., Correia-da-Silva, J. (2017), "Agglomeration patterns in a multi-regional economy without income effects", Economic Theory, forthcoming, https://doi.org/10.1007/s00199-017-1065-9.

Ikeda, K., Akamatsu, T., Kono, T. (2012), "Spatial period-doubling agglomeration of a core-periphery model with a system of cities", Journal of Economic Dynamics and Control, 36(5), 754-778.

Ikeda, K., Murota, K., Akamatsu, T., Kono, T., Takayama, Y. (2014), "Self-organization of hexagonal agglomeration patterns in new economic geography models", Journal of Economic Behavior $\& 3$ Organization, 99, 32-52.

Krugman, P. (1991), "Increasing Returns and Economic Geography", Journal of Political Economy, 99 (3), 483-499.

Mossay, P. (2013), "A theory of rational spatial agglomerations", Regional Science and Urban Economics, 43 (2), 385-394. 
Ottaviano, G., Tabuchi, T., Thisse, J.-F. (2002), "Agglomeration and Trade Revisited", International Economic Review, 43 (2), 409-35.

Oyama, D. (2009), "Agglomeration under forward-looking expectations: Potentials and global stability", Regional Science and Urban Economics, 39 (6), 696-713.

Pflüger (2004), "A simple, analytically solvable, Chamberlinian agglomeration model", Regional Science and Urban Economics, 34, 565-573.

Picard, P. M., Tabuchi, T. (2010), "Self-organized agglomerations and transport costs", Economic Theory, 43 (2), 565-589.

Puga, D. (1999), "The Rise and Fall of Regional Inequalities", European Economic Review, $43,303-334$.

Robert-Nicoud, F. (2005), "The Structure of Simple 'New Economic Geography' Models (or, On Identical Twins)", Journal of Economic Geography, 5 (2), 201-234.

Tabuchi, T. (2014), "Historical trends of agglomeration to the capital region and new economic geography", Regional Science and Urban Economics, 44, 50-59.

Tabuchi, T., Thisse, J.-F. (2011), "A new economic geography model of central places", Journal of Urban Economics, 69 (2), 240-252.

Tabuchi, T., Thisse, J. F., Zeng, D. Z. (2005), "On the number and size of cities", Journal of Economic Geography, 5(4), 423-448.

Zeng, D. Z., Uchikawa, T. (2014), "Ubiquitous inequality: the home market effect in a multicountry space", Journal of Mathematical Economics, 50, 225-233. 


\section{Appendix A - Nominal wage in the 3-region model}

After (12) and (13), for $n=3$, we have the following linear system of equations:

$$
\left\{\begin{array}{l}
w_{1}\left(1-\frac{\mu}{\sigma} \frac{H_{1}}{R_{1}}\right)-w_{2}\left(\frac{\mu}{\sigma} \frac{\phi H_{2}}{R_{2}}\right)-w_{3}\left(\frac{\mu}{\sigma} \frac{\phi H_{3}}{R_{3}}\right)=\frac{\mu}{\sigma} \frac{L}{3}\left(\frac{1}{R_{1}}+\frac{\phi}{R_{2}}+\frac{\phi}{R_{3}}\right) \\
w_{1}\left(-\frac{\mu}{\sigma} \frac{\phi H_{1}}{R_{1}}\right)+w_{2}\left(1-\frac{\mu}{\sigma} \frac{H_{2}}{R_{2}}\right)-w_{3}\left(\frac{\mu}{\sigma} \frac{\phi H_{3}}{R_{3}}\right)=\frac{\mu}{\sigma} \frac{L}{3}\left(\frac{\phi}{R_{1}}+\frac{1}{R_{2}}+\frac{\phi}{R_{3}}\right) \\
w_{1}\left(-\frac{\mu}{\sigma} \frac{\phi H_{1}}{R_{1}}\right)-w_{2}\left(\frac{\mu}{\sigma} \frac{\phi H_{2}}{R_{2}}\right)+w_{3}\left(1-\frac{\mu}{\sigma} \frac{\phi H_{3}}{R_{3}}\right)=\frac{\mu}{\sigma} \frac{L}{3}\left(\frac{\phi}{R_{1}}+\frac{\phi}{R_{2}}+\frac{1}{R_{3}}\right)
\end{array}\right.
$$

where $R_{j} \equiv \sum_{m=1}^{3} \phi_{m j} H_{m}$. The solution to this system is of the following form:

$$
w_{i}=\frac{D w_{i}}{D}
$$

where the denominator $D$ stands for the determinant of matrix $A$ and $D w_{i}$ is the determinant of the matrix obtained by replacing the $i$-th column of $A$ by the column vector $B$. We have:

$$
D=1-\frac{\mu}{\sigma} \sum_{j=1}^{3} \frac{H_{j}}{R_{j}}+\frac{\mu^{2}}{\sigma^{2}}\left(1-\phi^{2}\right)\left(\frac{H_{1} H_{2}}{R_{1} R_{2}}+\frac{H_{1} H_{3}}{R_{1} R_{3}}+\frac{H_{2} H_{3}}{R_{2} R_{3}}\right)-\frac{\mu^{3}}{\sigma^{3}}\left(2 \phi^{3}-3 \phi^{2}+1\right) \prod_{j=1}^{3} \frac{H_{j}}{R_{j}} .
$$

For $i=1, D w_{1}$ is given by:

$$
\begin{aligned}
D w_{1} & =\frac{\mu}{\sigma} \frac{L}{3}\left\{\left(\sum_{j=1}^{3} \frac{\phi_{1 j}}{R_{j}}\right)+\frac{\mu}{\sigma}\left[\phi(\phi-1) \frac{H_{2}+H_{3}}{R_{2} R_{3}}+\frac{\phi^{2}-1}{R_{1}}\left(\frac{H_{2}}{R_{2}}+\frac{H_{3}}{R_{3}}\right)\right]+\right. \\
& \left.+\frac{\mu^{2}}{\sigma^{2}}\left(2 \phi^{3}-3 \phi^{2}+1\right) \frac{H_{2} H_{3}}{R_{1} R_{2} R_{3}}\right\}
\end{aligned}
$$

The expression for the nominal wage in region 1 is:

$$
w_{1}=\frac{\frac{\mu}{\sigma} \frac{L}{3}\left\{\sum_{j=1}^{3} \frac{\phi_{1 j}}{R_{j}}+\frac{\mu}{\sigma}\left[\phi(\phi-1) \frac{H_{2}+H_{3}}{R_{2} R_{3}}+\frac{\phi^{2}-1}{R_{1}}\left(\frac{H_{2}}{R_{2}}+\frac{H_{3}}{R_{3}}\right)\right]+\frac{\mu^{2}}{\sigma^{2}}\left(2 \phi^{3}-3 \phi^{2}+1\right) \frac{H_{2} H_{3}}{R_{1} R_{2} R_{3}}\right\}}{1-\frac{\mu}{\sigma} \sum_{j=1}^{3} \frac{H_{j}}{R_{j}}+\frac{\mu^{2}}{\sigma^{2}}\left(1-\phi^{2}\right)\left(\frac{H_{1} H_{2}}{R_{1} R_{2}}+\frac{H_{1} H_{3}}{R_{1} R_{3}}+\frac{H_{2} H_{3}}{R_{2} R_{3}}\right)-\frac{\mu^{3}}{\sigma^{3}}\left(2 \phi^{3}-3 \phi^{2}+1\right) \prod_{j=1}^{3} \frac{H_{j}}{R_{j}}} .
$$

Generically,

$$
w_{i}=\frac{\frac{\mu}{\sigma} \frac{L}{3}\left\{\sum_{j=1}^{3} \frac{\phi_{i j}}{R_{j}}+\frac{\mu}{\sigma}\left[\phi(\phi-1) \frac{\sum_{k \neq i} H_{k}}{\prod_{k \neq i} R_{k}}+\frac{\phi^{2}-1}{R_{i}} \sum_{k \neq i} \frac{H_{k}}{R_{k}}\right]+\frac{\mu^{2}}{\sigma^{2}}\left(2 \phi^{3}-3 \phi^{2}+1\right) \frac{1}{R_{i}} \prod_{k \neq i} \frac{H_{k}}{R_{k}}\right\}}{1-\frac{\mu}{\sigma} \sum_{j=1}^{3} \frac{H_{j}}{R_{j}}+\frac{\mu^{2}}{\sigma^{2}}\left(1-\phi^{2}\right)\left(\frac{H_{1} H_{2}}{R_{1} R_{2}}+\frac{H_{1} H_{3}}{R_{1} R_{3}}+\frac{H_{2} H_{3}}{R_{2} R_{3}}\right)-\frac{\mu^{3}}{\sigma^{3}}\left(2 \phi^{3}-3 \phi^{2}+1\right) \prod_{j=1}^{3} \frac{H_{j}}{R_{j}}} .
$$




\section{Appendix B - Jacobian and total dispersion}

As a prerequisite to Proposition 1, establishing the stability of total dispersion, we have the following Lemma.

Lemma 1. Concerning total dispersion: (i) The Jacobian matrix of the (n-1)-dimensional system in (18) at total dispersion has a repeated real eigenvalue with multiplicity $n-1$ given by:

$$
\frac{\partial \omega_{i}}{\partial h_{i}}\left(\frac{1}{n}, \frac{1}{n}, \ldots, \frac{1}{n}\right)
$$

Proof. In this proof, we display all $n$ region coordinates of the real wage function rather than omit the last coordinate, as this clarifies the way variations in the first $n-1$ coordinates are reflected in $h_{n}=\sum_{i=1}^{n-1} h_{i}$. Changes in $h_{i}$ are symmetrically reflected in $h_{n}$, i.e., for any $i \neq n$, a small variation $\Delta h_{i}=\varepsilon$ implies that $\Delta h_{n}=-\varepsilon$. An element of the main diagonal of the Jacobian matrix is given by:

$$
J_{i i}=\frac{\partial \omega_{i}}{\partial h_{i}}-\frac{\partial \bar{\omega}}{\partial h_{i}}
$$

We will first show that $\partial_{h_{i}} \bar{\omega}=0, \forall i$ at total dispersion, i.e., $J_{i i}=\frac{\partial \omega_{i}}{\partial h_{i}}$.

Assume, by way of contradiction, that $\partial_{h_{1}} \bar{\omega}>0$. Then $\bar{\omega}\left(\frac{1}{n}+\varepsilon, \frac{1}{n}, \ldots ., \frac{1}{n}, \frac{1}{n}-\varepsilon\right)>$ $\bar{\omega}\left(\frac{1}{n}, \frac{1}{n}, \ldots, \frac{1}{n}\right)>\bar{\omega}\left(\frac{1}{n}-\varepsilon, \frac{1}{n}, \ldots, \frac{1}{n}, \frac{1}{n}+\varepsilon\right)$. However, the fact that regions are completely symmetric in all respects asserts that $\bar{\omega}$ is invariant in the permutation of any $h_{i}$ and $h_{j}, i \neq j$, and thus also $h_{1}$ and $h_{n}$. Hence, $\bar{\omega}\left(\frac{1}{n}+\varepsilon, \frac{1}{n}, \ldots ., \frac{1}{n}, \frac{1}{n}-\varepsilon\right)=\bar{\omega}\left(\frac{1}{n}-\varepsilon, \frac{1}{n}, \ldots ., \frac{1}{n}, \frac{1}{n}+\varepsilon\right)$, and we arrive at a contradiction. As a result, it must be that $\partial_{h_{1}} \bar{\omega}=0$ and therefore $J_{11}=$ $\partial_{h_{1}} \omega_{1}$. Moreover, symmetry between regions implies that $\partial_{h_{1}} \omega_{1}=\partial_{h_{2}} \omega_{2}=\ldots=\partial_{h_{n-1}} \omega_{n-1}$ at total dispersion. This leads to the conclusion that $J_{11}=J_{22}=\ldots=J_{n-1, n-1}$, implying that all diagonal elements of the Jacobian are given by $J_{i i}=\partial_{h_{i}} \omega_{i}$.

We will next show that all elements outside the main diagonal are zero at total dispersion. An element outside the main diagonal is given by:

$$
J_{i j}=\frac{\partial \omega_{i}}{\partial h_{j}}-\frac{\partial \bar{\omega}}{\partial h_{j}}
$$

We have seen that $\partial_{h_{i}} \bar{\omega}=0$ at total dispersion. A similar argument to the one used to prove that $\partial_{h_{i}} \bar{\omega}=0$ shows that $\partial_{h_{j}} \omega_{i}=0$ at total dispersion. Thus, all off-diagonal elements are zero and the $(n-1) \times(n-1)$ Jacobian matrix at total dispersion is a scalar multiple of the identity,

$$
\frac{\partial \omega_{i}}{\partial h_{i}}\left(\frac{1}{n}, \frac{1}{n}, \ldots, \frac{1}{n}\right) I
$$




\section{Proof of Proposition 1}

We compute $\partial w_{1} / \partial h_{1}$ at total dispersion while avoiding having to solve a system of $n-1$ linear equations using just two wage equations from (15), e.g., for $w_{1}$ and $w_{n}$. The reason is that, after Lemma 1 , there are $n-2$ wages equal to $w_{j}$ for which $\partial w_{j} / \partial h_{1}=0$. As a result, we still have $w_{1}$ and $w_{n}$ that depend on $h_{1}$. We have:

$$
\left\{\begin{array}{l}
w_{1}\left(1-\frac{\mu}{\sigma} \frac{h_{1}}{r_{1}}\right)-(n-2) w_{j} \frac{\mu}{\sigma} \phi\left(\frac{1 / n}{r_{j}}\right)-w_{n}\left(\frac{\mu}{\sigma} \phi \frac{\frac{2}{n}-h_{1}}{r_{n}}\right)=\frac{1}{H(n)} \frac{\mu}{\sigma} \frac{L(n)}{n}\left(\frac{1}{r_{1}}+(n-2) \frac{\phi}{r_{j}}+\frac{\phi}{r_{n}}\right) \\
-w_{1}\left(\frac{\mu}{\sigma} \phi \frac{h_{1}}{r_{1}}\right)-(n-2) w_{j} \frac{\mu}{\sigma} \phi\left(\frac{1 / n}{r_{j}}\right)+w_{n}\left(1-\frac{\mu}{\sigma} \frac{\frac{2}{n}-h_{1}}{r_{n}}\right)=\frac{1}{H(n)} \frac{\mu}{\sigma} \frac{L(n)}{n}\left(\frac{\phi}{r_{1}}+(n-2) \frac{\phi}{r_{j}}+\frac{1}{r_{n}}\right)
\end{array}\right.
$$

where $r_{1}=h_{1}+\phi \frac{n-2}{n}+\phi\left(\frac{2}{n}-h_{1}\right), r_{j}=\frac{1}{n}+\frac{(n-1) \phi}{n}$ and $r_{n}=h_{1} \phi-h_{1}+\frac{(n-2) \phi}{n}+\frac{2}{n}$. Solving the second equation for $w_{n}$ and replacing it in the first one, knowing that $\partial w_{j} / \partial h_{1}=0$ at total dispersion, we reach

$$
\frac{\partial w_{1}}{\partial h_{1}}\left(\frac{1}{n}, \frac{1}{n}, \ldots, \frac{1}{n}\right)=\frac{\mu L(n) n(\phi-1)[\mu+\mu(n-1) \phi-\sigma(1-\phi)]}{H(n)(\mu-\sigma)[(n-1) \phi+1][-\mu(1-\phi)+(n-1) \sigma \phi+\sigma]} .
$$

By (14), we have:

$$
\frac{\partial P_{1}^{\mu} / \partial h_{1}}{P_{1}^{\mu}}=\frac{\mu n(\phi-1)}{(\sigma-1)[(n-1) \phi+1]} .
$$

We can then compute the derivative of the real wage in region 1 with respect to $h_{1}$ :

$$
\frac{\partial \omega_{1}}{\partial h_{1}}\left(\frac{1}{n}, \frac{1}{n}, \ldots, \frac{1}{n}\right)=\frac{\mu L(n) n(\phi-1)\left\{\mu^{2}(\phi-1)+\mu(2 \sigma-1)[(n-1) \phi+1]+(\sigma-1) \sigma(\phi-1)\right\}}{H(n)(\sigma-1)(\mu-\sigma)[(n-1) \phi+1][\mu+(n-1) \sigma \phi+\sigma-\mu]} .
$$

Note that the denominator is negative as is the factor $(\phi-1)$ in the numerator. Hence, total dispersion is stable if:

$$
B P(\phi) \equiv \mu(2 \sigma-1)[(n-1) \phi+1]-\mu^{2}(1-\phi)-(\sigma-1) \sigma(1-\phi)<0,
$$

which concludes the proof.

\section{Appendix C - Jacobian and partial dispersion}

The next result is used in the study of partial dispersion (also holds for total dispersion).

Lemma 2. Configurations $h=\left(h_{1}, h_{2}, \ldots, h_{n}\right)$ such that $h_{1}=h_{n}=a \in(0,1)$ and $h_{2}=$ $\ldots=h_{n-1}=b \in[0,1)$ satisfy $\frac{\partial \bar{\omega}}{\partial h_{1}}=0$. 
Proof. Suppose that $\frac{\partial \bar{\omega}}{\partial h_{1}}(a, b, \ldots, b, a)>0$. Then:

$$
\bar{\omega}(a+\varepsilon, b, \ldots, b, a-\varepsilon)>\bar{\omega}(a, b, \ldots, b, a) .
$$

But, $\bar{\omega}$ is invariant by the permutation that interchanges identically populated regions and therefore:

$$
\bar{\omega}(a-\varepsilon, b, \ldots, b, a+\varepsilon)>\bar{\omega}(a, b, \ldots, b, a)
$$

indicating that $\frac{\partial \bar{\omega}}{\partial h_{1}}<0$, which contradicts the assumption. Therefore, we have $\frac{\partial \bar{\omega}}{\partial h_{1}}=0$, which concludes the proof.

\section{Proof of Proposition 3}

(i). First, since $h_{j}=0$ for $j=\{2, \ldots, n-1\}$, we have the following condition for stability of partial dispersion:

$$
\omega_{1}>\omega_{j}
$$

Second, we need to ensure not only that $h_{j}$ will remain zero but also that both $h_{1}$ and $h_{n}$ will remain at $\frac{1}{2}$. If any of the skilled workers migrates from region 1 to region $n$, we need them to want to return to region 1 (symmetry implies the same in the opposite direction). This is achieved when an increase in $h_{1}$ leads to a decrease in the difference between the real wage $\omega_{1}$ and the real wage average $\bar{\omega}$ :

$$
\frac{\partial \omega_{1}}{\partial h_{1}}\left(\frac{1}{2}, 0, \ldots, 0, \frac{1}{2}\right)-\frac{\partial \bar{\omega}}{\partial h_{1}}\left(\frac{1}{2}, 0, \ldots, 0, \frac{1}{2}\right)<0 .
$$

Lemma 2 asserts that $\frac{\partial \bar{\omega}}{\partial h_{1}}\left(\frac{1}{2}, 0, \ldots, 0, \frac{1}{2}\right)=0$. As a result, the inequality reduces to:

$$
\frac{\partial \omega_{1}}{\partial h_{1}}\left(h^{p}\right)<0
$$

Notice that both conditions are sufficient for stability, but need not be necessary. If one of the conditions (or both) holds in equality, partial dispersion may also be stable. Therefore, what we can say is that if partial dispersion is stable, then:

$$
\omega_{i}\left(h^{p}\right) \geq \omega_{j}\left(h^{p}\right) \text { and } \frac{\partial \omega_{i}}{\partial h_{i}}\left(h^{p}\right) \leq 0, i=\{1, n\}, \forall j \neq i
$$

where $h^{p}=\left(\frac{1}{2}, 0, \ldots, 0, \frac{1}{2}\right)$.

(ii). We now proceed to compute $\omega_{i}\left(h^{p}\right)$ and and $\frac{\partial \omega_{i}}{\partial h_{i}}\left(h^{p}\right)$. First, we determine the nominal wages in regions 1 and $n$ at partial dispersion. For regions 1 and $n$ we use 
equation (15) to get:

$$
w_{1}\left(h^{p}\right)=w_{n}\left(h^{p}\right)=\frac{\mu L(n)}{H(n)(\sigma-\mu)} .
$$

As for the other $j$ regions we have:

$$
w_{j}\left(h^{p}\right)=\frac{\mu L(n)\left[n\left(\frac{2 \mu \phi^{2}}{\sigma-\mu}+\phi+1\right)-2(1-\phi)(2 \phi+1)\right]}{H(n) n \sigma \phi(\phi+1)} .
$$

Then, the first condition for stability of partial dispersion requires:

$$
\frac{w_{1}}{w_{j}}>\left(\frac{P_{1}}{P_{j}}\right)^{\mu}
$$

which yields:

$$
\mu(n-2)(\phi-1)(2 \phi+1)+\sigma\left[2(\phi-1)(2 \phi+1)-n(\phi+1)\left(\phi 2^{\frac{\mu}{1-\sigma}}\left(\frac{\phi}{\phi+1}\right)^{\frac{\mu}{1-\sigma}}-1\right)\right]<0 .
$$

Rewriting it, we obtain:

$$
\gamma \equiv \sigma(1+\phi)\left[1-\phi^{1-\frac{\mu}{\sigma-1}}\left(\frac{1+\phi}{2}\right)^{\frac{\mu}{\sigma-1}}\right] n-[2 \sigma+\mu(n-2)](1-\phi)(2 \phi+1)<0 .
$$

In order to determine the second condition, we follow an approach analogous to that of the proof of Proposition 1 in Appendix B, selecting two wage equations from (15) and using Lemma 1 to state that only $w_{1}$ and $w_{n}$ depend on $h_{1}$ :

$$
\left\{\begin{array}{l}
w_{1}\left(1-\frac{\mu}{\sigma} \frac{h_{1}}{h_{1}+\phi\left(1-h_{1}\right)}\right)-w_{n}\left(\frac{\mu}{\sigma} \frac{\phi\left(1-h_{1}\right)}{\phi h_{1}+1-h_{1}}\right)=\frac{\mu L(n)}{\sigma n H(n)}\left(\frac{1}{h_{1}+\phi\left(1-h_{1}\right)}+n-2+\frac{\phi}{\phi h_{1}+1-h_{1}}\right) \\
w_{n}\left(1-\frac{\mu}{\sigma} \frac{1-h_{1}}{\phi h_{1}+1-h_{1}}\right)-w_{1}\left(\frac{\mu}{\sigma} \frac{\phi h_{1}}{h_{1}+\phi\left(1-h_{1}\right)}\right)=\frac{\mu L(n)}{\sigma n H(n)}\left(\frac{\phi}{h_{1}+\phi\left(1-h_{1}\right)}+n-2+\frac{1}{\phi h_{1}+1-h_{1}}\right)
\end{array}\right.
$$

Solving the second equation for $w_{n}$ and replacing in the first we obtain $w_{1}$ as a function of $h_{1}$. Then, differentiating with respect to $h_{1}$ and evaluating at $h_{1}=1 / 2$, we get:

$$
\frac{\partial w_{1}}{\partial h_{1}}\left(h^{p}\right)=\frac{4 \mu L(n)(\phi-1)[\mu+\mu(n-1) \phi+\sigma(\phi-1)]}{H(n) n(\phi+1)(\mu-\sigma)[\phi(\mu+\sigma)-\mu+\sigma]} .
$$

Differentiating the price index, by (14):

$$
\frac{\partial P_{1}^{\mu} / \partial h_{1}}{P_{1}^{\mu}}\left(h^{p}\right)=\frac{2 \mu(\phi-1)}{(\sigma-1)(\phi+1)} .
$$

Finally, we can compute the derivative of the real wage in region 1 with respect to $h_{1}$ at 
partial dispersion:

$$
\frac{\partial \omega_{1}}{\partial h_{1}}\left(h^{p}\right)=\frac{2(\phi-1)\left\{-\frac{\mu}{\sigma-1}-\frac{2[\mu+\mu(n-1) \phi+\sigma(\phi-1)]}{n[\phi(\mu+\sigma)-\mu+\sigma]}\right\}}{\phi+1}
$$

which is negative if and only if:

$$
\Psi \equiv \mu^{2} n(\phi-1)+\mu[\sigma(3 n \phi+n-2 \phi+2)-2 n \phi+2 \phi-2]+2(\sigma-1) \sigma(\phi-1)<0,
$$

which gives us the second condition, concluding the proof.

\section{Appendix D - Comparing models}

\section{Proof of Proposition 4}

The derivative of $S P$ in (23) with respect to $n$ is negative. This implies that an increase in the number of regions decreases $\phi_{s}$, for any given values of the remaining parameters. This shows that agglomeration is more likely in a FE model with more regions.

In the $n$-region model, the core-periphery price index ratio equals:

$$
\left(\frac{P_{i}}{P_{j}}\right)^{\mu}=\phi^{\frac{\mu}{\sigma-1}}
$$

which does not depend on the number of regions and proves part (i) of the proposition. The nominal wage in the core region $i$ is given by:

$$
w_{i}=\frac{\mu L(n)}{(\sigma-\mu) H(n)}
$$

The peripheral nominal wage is equal to:

$$
w_{j}=\frac{\mu L(n)\{\mu(\phi-1)[(n-1) \phi+1]+\sigma \phi(n+\phi-2)+\sigma\}}{H(n) n \sigma \phi(\sigma-\mu)}
$$

The wage ratio is given by:

$$
\frac{w_{i}}{w_{j}}=\frac{n \sigma}{\mu(\phi-1)[(n-1) \phi+1]+\sigma \phi(n+\phi-2)+\sigma},
$$

which one can check to be increasing in $n$, as:

$$
\frac{\partial\left(w_{i} / w_{j}\right)}{\partial n}=\frac{\sigma(\phi-1)^{2}(\sigma-\mu)}{\left\{\mu(\phi-1)[(n-1) \phi+1]+\sigma \phi(n+\phi-2)+\sigma^{2}\right\}}>0,
$$

thus concluding the final part of the proof. 


\section{Appendix E - Stability of agglomeration under asymmetries}

\section{Proof of Proposition 6}

At concentration in region 1 , we have, after (15):

$$
w_{1}=\frac{\mu L(n)}{H(n)(\sigma-\mu)}
$$

The wage in region 2 is given by:

$$
w_{2}=\frac{\mu\left\{L_{1} \phi^{2}+L_{2}\left[\sigma-\mu\left(1-\phi^{2}\right)\right]+\sum_{j=3}^{n} L_{j} \phi[\sigma-\mu(1-\phi)]\right\}}{H(n) \sigma \phi(\sigma-\mu)} .
$$

Recalling the discussion in Section 3.2, stability of agglomeration is given by $w_{1} \phi^{\frac{\mu}{\sigma-1}}-w_{2}<$ 0 , yielding:

$$
\mu \frac{\sigma \phi L(n)-\phi^{\frac{\mu}{\sigma-1}}\left\{\sigma \phi^{2} L_{1}+\phi \sum_{j=3}^{n} L_{j}[\sigma-\mu(1-\phi)]+L_{m}\left[\sigma-\mu\left(1-\phi^{2}\right)\right]\right\}}{H(n) \sigma \phi(\mu-\sigma)}<0,
$$

which, given that $\mu-\sigma<0$, becomes:

$$
\Gamma \equiv \phi^{\frac{\mu}{\sigma-1}}\left\{\sigma \phi^{2} L_{1}+\phi \sum_{j=3}^{n} L_{j}[\sigma-\mu(1-\phi)]+L_{2}\left[\sigma-\mu\left(1-\phi^{2}\right)\right]\right\}-\sigma \phi L(n)<0 .
$$

The global endowment of farmers can be rewritten as $L(n)=\left(L_{1}+\sum_{j=3}^{n} L_{j}+L_{2}\right)$. Note that $\Gamma^{\prime \prime}(\phi)>0$, so $\Gamma(\phi)$ is convex. Moreover, $\Gamma(0)=L_{2}(\sigma-\mu)>0, \Gamma(1)=0$, and

$$
\Gamma^{\prime}(1)=\frac{\mu L(n)}{\sigma-1}+\mu\left(L_{1}+3 L_{2}+2 \sum_{j=3}^{n} L_{j}\right)+\sigma\left(L_{1}-L_{2}\right)
$$

If $L_{1} \geq L_{2}$ then $\Gamma^{\prime}(1)>0$ and there exists a single sustain point $\phi_{s} \equiv \phi \in(0,1)$ above which agglomeration in region 1 is stable. This concludes the proof. 